\title{
High Phylogenetic Utility of an Ultraconserved Element Probe Set Designed for Arachnida
}

2
Running title: Utility of arachnid UCE probe set

James Starrett $^{1}$ *, Shahan Derkarabetian ${ }^{1,2 *}$, Marshal Hedin ${ }^{1}$, Robert W. Bryson Jr. ${ }^{3,4}$, John E. McCormack $^{4}$, Brant C. Faircloth ${ }^{5}$

1 Department of Biology, 5500 Campanile Drive, San Diego State University, San Diego, CA 92182, USA

2 Department of Biology, 900 University Avenue, University of California, Riverside, Riverside, CA 92521, USA

3 Department of Biology and Burke Museum of Natural History and Culture, University of

Washington, 4331 Memorial Way Northeast, Seattle, WA 98195, USA

4 Moore Laboratory of Zoology, Occidental College, Los Angeles, CA 90041, USA

5 Department of Biological Sciences and Museum of Natural Science, Louisiana State

University, Baton Rouge, LA 70803, USA

* equal contribution

\section{Corresponding author:}

Shahan Derkarabetian

Department of Biology

San Diego State University 5500 Campanile Dr.

San Diego, CA 92182-4614

Email: sderkarabetian@gmail.com 


\section{Abstract}

32 Arachnida is an ancient, diverse, and ecologically important animal group that contains a number

33 of species of interest for medical, agricultural, and engineering applications. Despite this applied

34 importance, many aspects of the arachnid tree of life remain unresolved, hindering comparative

35 approaches to arachnid biology. Biologists have made considerable efforts to resolve the

36 arachnid phylogeny; yet, limited and challenging morphological characters, as well as a dearth of

37 genetic resources, have confounded these attempts. Here, we present a genomic toolkit for

38 arachnids featuring hundreds of conserved DNA regions (ultraconserved elements or UCEs) that

39 allow targeted sequencing of any species in the arachnid tree of life. We used recently developed

40 capture probes designed from conserved genomic regions of available arachnid genomes to

41 enrich a sample of loci from 32 diverse arachnids. Sequence capture returned an average of 487

42 UCE loci for all species, with a range from 170 to 722 . Phylogenetic analysis of these UCEs

43 produced a highly resolved arachnid tree with relationships largely consistent with recent

44 transcriptome-based phylogenies. We also tested the phylogenetic informativeness of UCE

45 probes within the spider, scorpion, and harvestman orders, demonstrating the utility of these

46 markers at shallower taxonomic scales, even down to the level of species differences. This probe

47 set will open the door to phylogenomic and population genomic studies across the arachnid tree

48 of life, enabling systematics, species delimitation, species discovery, and conservation of these

49 diverse arthropods.

51 Key words: phylogenomics, target enrichment, ultraconserved elements, arthropods, Arachnida 


\section{Introduction}

Arachnida is an extremely ancient and diverse arthropod lineage, including conspicuous taxa such as spiders, scorpions, mites, and ticks. The oldest known arachnid fossils consist mostly of scorpions and extinct trigonotarbids from the Silurian period, 443-416 million years ago (MYA; Laurie 1899; Jeram et al. 1990; Dunlop, 1996; Dunlop et al. 2008). More than 110,000 species of arachnids have been described, with spiders and mites ranking among the most diverse of all animal orders (Harvey 2002; Zhang 2011). Yet, more than half of arachnid species diversity remains to be discovered (Chapman 2009). Arachnida also contains a number

60 of medically important venomous or disease-vector species and many important agricultural mite

61 pests, while spiders are of particular interest to biologists and engineers for the strong and elastic

62 silk fibers they produce. Despite the attention arachnids have received for their ecological

63 importance and practical utility to humans, phylogenetic relationships among and within many

64 arachnid orders remain uncertain. At the root of this problem is that morphological characters are

65 limited and difficult to interpret (Shultz 2007), and genomic resources for many species in this

66 group are sparse. Adding to the difficulty is the uncertainty in the rooting of the arachnid tree,

67 with fossil, morphological, and molecular data recovering drastic discrepancies in early arachnid

68 relationships and placing traditional non-arachnid chelicerates within Arachnida (Wheeler and

69 Hayashi 1998; Masta et al. 2009; Regier et al. 2010; Sharma et al. 2014). Genome wide

70 phylogenetic markers are essential for resolving deep relationships within Arachnida and for

71 helping to uncover the numerous arachnid species that await discovery.

72 Ultraconserved elements (UCEs) provide one potential target for a universal set of

73 genomic markers for arachnids and would allow researchers to collect genomic information from

74 diverse taxa across the arachnid tree of life. UCEs are segments of DNA that are highly 
conserved across divergent taxa (Bejerano et al. 2004) and are thought regulate and/or enhance gene expression (Alexander et al. 2010). UCEs can also be used as anchors to target and retrieve

77 variable DNA sequence flanking the core UCE regions, and the flanking DNA shows a trend of

78 increasing genetic variability as distance from the core UCE increases (Faircloth et al. 2012).

79 UCEs are ideal markers for molecular systematics for several reasons. Whereas transcriptomes

80 require high-quality RNA as input, the UCE protocol only requires DNA, and enrichments can

81 be performed using relatively low starting DNA concentrations. This allows the method to be

82 extended to small-bodied taxa, large collections of specimens preserved for Sanger-based DNA

83 research, and even to "standard" museum specimens with varying levels of DNA degradation

84 (McCormack et al. 2015). Homology between UCE loci across divergent taxa is also easy to

85 assess because the core UCE region often displays $>95 \%$ sequence similarity, and UCE cores are

86 rarely duplicated or paralogs (Derti et al. 2006). Although core UCE regions show reduced

87 sequence variation, UCE-flanking DNA shows levels of phylogenetic informativeness equal to

88 or greater than that of traditionally used protein-coding markers (Gilbert et al. 2015).

UCEs have been successfully used in phylogenetic studies at multiple taxonomic levels,

90 including shallow, young divergences ( 5 MYA, within species; Smith et al. 2014; Harvey et al.

91 2016; Manthey et al. 2016) and deep, ancient divergences (e.g., all amniotes; Faircloth et al.

92 2012). Originally developed for use in tetrapods, the vast majority of UCE phylogenetic studies

93 have been conducted on vertebrate taxa including all amniotes (Faircloth et al. 2012), mammals

94 (McCormack et al. 2012), birds (McCormack et al. 2013; Smith et al. 2014), reptiles (Crawford

95 et al. 2012; Crawford et al. 2015), and fish (Faircloth et al. 2013). More recently, UCE probe

96 sets have been developed for use in arthropod taxa, including Hymenoptera, Coleoptera, Diptera,

97 Lepidoptera, and Hemiptera (Faircloth et al. 2015; Faircloth 2016). 
Here, we perform an in vitro test of a recently developed bait set targeting arachnid UCEs

99 (Faircloth 2016). We then demonstrate the phylogenetic utility of this probe set at multiple

100 evolutionary timescales, from those spanning hundreds of millions of years to species-level

101 divergences of $<10$ million years. We reconstruct well-supported phylogenies between and

102 within arachnid orders, and we demonstrate that these UCE baits may also be useful when

103 reconstructing species level relationships.

104 Materials and Methods

\section{UCE library construction and enrichment}

UCE capture was tested using the master arachnid bait set (Faircloth 2016) on 32

107 arachnid samples representing six orders (Table 1). The orders selected span the root of

108 Arachnida (Regier et al. 2010; Sharma et al. 2014). Within three orders (Araneae, Opiliones,

109 Scorpiones), taxa were selected from major lineages, including across the internal root for each

110 (Hedin et al. 2012; Bond et al. 2014; Sharma et al. 2015). To assess UCE variability between

111 closely related taxa, we included two turret spiders from the Antrodiaetus riversi complex (Hedin

112 et al. 2013), two harvestmen representing Briggsus pacificus and B. bilobatus, and a second bark

113 scorpion Centruroides sculpturatus to complement the published C. exilicauda genome. Voucher

114 specimens are located in the San Diego State University Terrestrial Arthropod Collection

115 (SDSU_TAC). In the case of small arachnids (e.g., mites and ticks), whole specimens were used

116 in extractions, but vouchers from the same locality are deposited in SDSU_TAC.

117 Genomic DNA was extracted from legs or whole specimens using the DNeasy Blood and

118 Tissue Kit (Qiagen, Valencia, CA) following the manufacturer's protocol. DNA concentrations

119 were determined with a Qubit fluorometer (Life Technologies, Inc.) and run out on a 1\% agarose 
120 gel to assess quality. The lowest starting DNA quantity for processing was $108 \mathrm{ng}$ (Siro

121 acaroides, a small-bodied harvestmen typically $<2 \mathrm{~mm}$ in length), while most samples started

122 with approximately $500 \mathrm{ng}$ (Table 1). Samples with high molecular weight DNA were

123 fragmented with a QSonica Q800R sonicator for 10-12 cycles of $20 \mathrm{sec}$ on/20 sec off, resulting

124 in fragments predominantly in the range of 300-1000 bp. DNA from sample AR014 (Neomolgus

125 littoralis) was partially degraded and not sonicated.

127 generic SPRI substitute used for bead clean-up steps (Rohland and Reich 2012; Glenn et al.

128 2016). Universal adapters were ligated onto end-repaired and A-tailed DNA fragments. Each

129 adapter-ligated library was amplified in a $50 \mu 1$ total reaction, which consisted of $15 \mu 1$ of adapter

130 ligated DNA, 1X KAPA HiFi HotStart ReadyMix, and $5 \mu \mathrm{M}$ of each Illumina TruSeq dual-

131 indexed primer (i5 and i7) with modified 8 bp indexes (Glenn et al. 2016). Amplification

132 conditions were $98^{\circ} \mathrm{C}$ for $45 \mathrm{sec}$, followed by $10-12$ cycles of $98^{\circ} \mathrm{C}$ for $15 \mathrm{sec}, 60^{\circ} \mathrm{C}$ for $30 \mathrm{sec}$,

133 and $72^{\circ} \mathrm{C}$ for $60 \mathrm{sec}$, and then a final extension of $72^{\circ} \mathrm{C}$ for $60 \mathrm{sec}$. After bead clean-up of

134 amplified libraries, equimolar amounts of libraries were combined into $1000 \mathrm{ng}$ total pools. Pool

135 combinations ranged from 1-8 individual libraries (Table 1). Due to a low concentration

136 following library preparation and amplification, the pool containing sample AR014 (Neomolgus

137 littoralis) included a lower amount of library (108.9 ng) compared to the other two libraries that 138 we included (333 ng each).

139 Target enrichment of libraries was performed using the MySelect kit (Microarray)

140 following the Target Enrichment of Illumina Libraries v. 1.5 protocol

141 (http://ultraconserved.org/\#protocols). Custom TruSeq adaptor blockers (Glenn et al. 2016) and

142 standard MYbaits blockers were annealed to $147 \mathrm{ng} / \mathrm{uL}$ library pools, followed by hybridization 
143 to the master arachnid bait set (Faircloth 2016). Hybridizations were performed at $65^{\circ} \mathrm{C}$ for 24

144 hours. After hybridization, library pools were bound to Dynabeads MyOne Streptavidin C1

145 magnetic beads (Life Technologies) for enrichment. We performed with-bead PCR recovery of

146 the post-hybridization enrichments in a $50 \mu \mathrm{l}$ volume reaction consisting of $15 \mu$ l enriched DNA,

147 1X KAPA HiFi HotStart ReadyMix, and $5 \mu \mathrm{M}$ each of TruSeq Forward and Reverse primers.

148 Amplification conditions were $98^{\circ} \mathrm{C}$ for $45 \mathrm{sec}$, followed by 18 cycles of $98^{\circ} \mathrm{C}$ for $15 \mathrm{sec}, 60^{\circ} \mathrm{C}$

149 for $30 \mathrm{sec}$, and $72^{\circ} \mathrm{C}$ for $60 \mathrm{sec}$, and then a final extension of $72^{\circ} \mathrm{C}$ for five minutes. Following

150 PCR recovery, libraries were quantified using a Qubit fluorometer and diluted to $5 \mathrm{ng} / \mu \mathrm{L}$. We

151 performed qPCR quantification of enriched library pools, and we prepared a $10 \mathrm{uM}$ mix of each

152 pool at equimolar ratios. We sequenced the library pool using a partial run of paired-end $150 \mathrm{bp}$

153 sequencing on an Illumina NextSeq (Georgia Genomics Facility).

Read Processing, Contig Assembly, and Matrix Creation

Raw read data were processed using the PHYLUCE pipeline (Faircloth 2015). Adapter

156 removal and quality control trimming were done using the Illumiprocessor wrapper (Faircloth

157 2013) using default values. Reads were assembled using Trinity version r2013-02-25 (Grabherr

158 et al. 2011). Contigs from all samples were matched to probes using minimum coverage and

159 minimum identity values of 65 . We additionally extracted UCE loci in silico from available

160 arachnid genomes and Limulus polyphemus. UCE loci were aligned using mafft (Katoh and

161 Standley, 2013) and trimmed with gblocks (Castresana 2000; Talavera and Castresana 2007) as

162 implemented in the PHYLUCE pipeline. Multiple datasets were created for downstream

163 analyses. First, a "2perArachnid" dataset was created that contained up to two representative

164 samples from each arachnid order sequenced, plus Limulus as an outgroup. This included UCE

165 data extracted from both novel samples and previously deposited genomes. Representative 
166 samples were chosen to span the root node of the relevant arachnid orders and based on number

167 of UCE loci recovered. Because the purpose of our study was not to reconstruct arachnid

168 phylogeny, we did not include all arachnid orders. Second, we assembled a "UCEsample"

169 dataset that included only samples newly sequenced for this study. Third, three individual

170 datasets were created that included all samples from within the orders Araneae, Opiliones, and

171 Scorpiones. For each of these individual-order datasets, two matrices were created, one including

172 the amblypygid Damon as the outgroup, and a second without an outgroup. Data matrices

173 without outgroups were used for determining matrix statistics, with the number of parsimony

174 informative characters computed using PAUP* 4.0 (Sinauer Associates, Inc.). Finally, to assess

175 species-level utility of UCEs, three congeneric datasets were created for Antrodiaetus (turret

176 spiders), Briggsus (Briggs' harvestmen), and Centruroides (bark scorpions).

177 Phylogenetic Analysis

178 Data matrices including an outgroup taxon were also subjected to phylogenetic analyses

179 using RAxML HPC v8.0 (Stamatakis 2014), implementing the rapid bootstrap algorithm

180 (Stamatakis et al. 2008) plus ML tree search option (-f a), 200 bootstrap replicates, and the

181 GTRGAMMA model. ML analyses were conducted on two matrices for each dataset, one with

$18270 \%$ taxon coverage for each locus and one with 50\% coverage. Topologies and support scores

183 based on $70 \%$ taxon coverage were nearly identical to those from $50 \%$ taxon coverage, and we

184 show matrix statistics and trees for only $50 \%$ datasets. All analyses were conducted on a late

$1852015 \mathrm{iMac}$ with a $4 \mathrm{GHz}$ Intel $\mathrm{i} 7$ processor, with the exception of contig assembly with Trinity,

186 which was run on a 12 core CentOS linux machine with 48 GB of RAM.

\section{Results}



average of 1,240,464 reads (94.2\%) passing quality control. Assemblies resulted in an average of

19235,884 contigs per sample. The number of UCE loci recovered from newly sequenced samples

193 varied between 170 and 722 (average=487), while the average recovered in silico from published 194 genomes was 675, including 555 UCEs from the outgroup Limulus. The average number of

195 recovered UCE loci differed (unpaired two tailed t-test, $\mathrm{t}=6.4308, \mathrm{df}=38, \mathrm{p}$-value $=<0.0001$ )

196 among orders, being higher in groups from which the probes were designed (Araneae,

197 Parasitiformes, Scorpiones: 605 loci) versus those that were not used in probe design

198 (Acariformes, Amblypygi, Opiliones, Thelyphonida: 376 loci). We tested whether the number of 199 samples included in a hybridization pool influenced the number of UCE loci recovered and 200 found no correlation $\left(\mathrm{R}^{2}=0.03\right)$, although the Opiliones-only pools recovered the fewest loci 201 (SuppFigure 1). Matrix statistics are presented for a 50\% taxon coverage dataset in Table 2. We 202 recovered identical topologies and nearly identical support scores for matrices with $70 \%$ taxon 203 coverage (not shown) despite an approximate 37\% decrease in loci numbers. Across the final 204 matrices, an average of 589 UCE loci were included in the 50\% matrices, the lowest being the 205 Opiliones + OUT datasets. Matrix lengths varied from 105,337 bp in the "UCEsample" matrix to $206452,309 \mathrm{bp}$ in the Scorpiones matrix. The average percentage of parsimony-informative 207 characters was $28.99 \%$.

208 For shallow time-scale comparisons, Briggsus (Briggs' harvestmen) resulted in the 209 lowest number of UCE loci recovered (Table 3; 292 loci, total length of 172,562, average locus 210 length 591), but contained the highest number of variable sites (25,524-14.8\%). Conversely, the 
211 Centruroides (bark scorpions) comparison recovered the highest number of UCE loci (585 loci,

212 total length of 583,454, average locus length 997.4) but lowest number of variable sites (7,660 -

213 1.3\%). The proportion of variable loci for all comparisons were above 0.9, with average number

214 of variable sites per locus ranging from 14-87 (Table 3).

215 Maximum-likelihood analyses demonstrated the utility of the arachnid-specific UCE

216 probe set in resolving relationships among and within arachnid orders (Figs. 1 - 3). For the

217 limited deep-level sample dataset (i.e., '2perArachnid'), bootstrap support was 100\% for all

218 nodes except the node uniting Scorpiones to Araneae + Pedipalpi (=Amblypygi + Thelyphonida)

219 (Fig. 1). For the Araneae dataset, most nodes were fully supported $(\mathrm{BS}=100 \%)$, while two nodes

220 within Entelegyne spiders had bootstrap values of 97\% (Fig. 2). Within Opiliones, all nodes

221 received bootstrap support $\geq 99 \%$ (Fig. 3), and within Scorpiones, all nodes were fully supported

222 except for one node with bootstrap support of 69\% (Fig. 3).

\section{Discussion}

224 Arachnid UCEs

225 The development of genetic markers for arachnids has lagged behind that of many groups due, in

226 part, to the ancient divergences within the group (at least 400 MYA; Rehm et al. 2012; Rota-

227 Stabelli et al. 2013) and the relatively few published arachnid genomic resources. Here, we

228 demonstrate the utility of a UCE probe set targeting around 1000 loci that successfully works for

229 all arachnid species tested (and likely also with close outgroups). Across all samples, which

230 includes the deepest divergence within Arachnida (Regier et al. 2010; Sharma et al. 2014), we

231 recovered $510 \mathrm{UCE}$ loci with more than 49,362 parsimony-informative sites. For datasets within

232 scorpions, spiders, and harvestmen, which each span root nodes estimated at hundreds of 
233 millions of years of divergence (Hedin et al. 2012; Bond et al. 2014; Sharma and Wheeler 2014),

234 we recovered 749, 724, and 381 loci, respectively. We did not find a significant correlation

235 between library pool size and average number of loci recovered (Fig. S1), and thus the lower

236 number of loci recovered for Opiliones likely reflects their distant relationship to the taxonomic

237 groups from which the probes were developed.

Phylogenetic Utility

Recent phylogenomic datasets based on transcriptomes have provided better-resolved and well-supported phylogenies compared to prior morphological and genetic work using few loci

241 (Hedin et al. 2012; Bond et al. 2014; Fernandez et al. 2014; Sharma et al. 2014; Sharma et al.

242 2015; Garrison et al. 2016). However, obtaining sequence coverage across taxa from

243 transcriptomes requires high-quality RNA and a consistent expression pattern, which may be

244 difficult to obtain for many non-model taxa. UCE sequence data have proven to be useful for

245 resolving relationships at both deep and shallow levels (Crawford et al. 2012; Faircloth et al.

246 2012; McCormack et al. 2012; Faircloth et al. 2013; McCormack et al. 2013; Smith et al. 2014;

247 Streicher et al. 2015; Meiklejohn et al. 2016), and can be obtained from museum specimens or 248 other potentially degraded samples (McCormack et al. 2015).

249 Our reconstructed phylogenies based on UCEs demonstrate the promising utility of this

250 probe set for collecting data to resolve relationships at a wide range of divergence levels, from

251 the deepest bifurcations in the arachnid tree to shallower divergences within genera. In our

252 limited deep level sample dataset (i.e., '2perArachnid'), we recovered well-supported

253 monophyletic groups for all orders for which more than one individual was included (Fig. 1).

254 Additionally, we obtained well-supported sister relationships between Thelyphonida and

255 Amblypygi (Pedipalpi), and a Pedipalpi sister group to Araneae, consistent with relationships 
256 based on morphology, Sanger sequence data, and transcriptome data (Giribet et al. 2002; Shultz

257 2007; Regier et al. 2010; Sharma et al. 2014). The low support in our phylogeny for the

258 placement of Scorpiones may be a result of our sparse sampling, or it may reflect the difficulty in

259 determining the position of this group due to a possible rapid radiation deep in the arachnid tree

260 of life (Sharma et al. 2014).

261 Phylogenetic analyses of the UCE data for our more densely sampled groups (Araneae,

262 Opiliones, Scorpiones; Figs. 2 and 3) also recover topologies that are mostly congruent with

263 recent transcriptome-based phylogenies (Hedin et al. 2012; Bond et al. 2014; Sharma et al. 2015;

264 Garrison et al. 2016). Within Araneae (Fig. 2), many well-supported relationships and clades are

265 recovered including Opisthothelae (Mygalomorphae + Araneomorphae), a split between the

266 Atypoidea and Avicularioidea within mygalomorphs, and a split between Haplogynae and

267 Entelegynae within araneomorphs. We also recover a well-supported Paleocribellate

268 (Hypochilus) + Filistatidae (Kukulcania) sister group, a relationship only recently hypothesized

269 based on transcriptome data (Bond et al. 2014). Relationships within Entelegynae using UCE

270 data were not consistent with those recovered with transcriptome data (Bond et al. 2014;

271 Garrison et al. 2016). However, there is disagreement in relationships between Araneoidea, RTA

272 spiders, and related taxa in the different transcriptome datasets, and support values are relatively

273 low at these nodes for both transcriptome and UCE data, indicating need for further sampling.

274 Within Opiliones (Fig. 3), we recover monophyletic Palpatores (Eupnoi + Dyspnoi) and

275 Laniatores, but we did not recover a monophyletic Phalangida (Palpatores + Laniatores) as

276 recovered with transcriptomes (Hedin et al. 2012; Sharma et al. 2014) and a five gene dataset

277 combined with morphological characters (Sharma and Giribet 2014). Within Scorpiones (Fig. 3),

278 phylogenetic analyses of UCEs recover the same split between parvorders Buthida and Iurida as 
279 inferred from analyses of transcriptomes (Sharma et al. 2015). However, the well-supported

280 position of Bothriurus deep within Iurida in our phylogeny, rather than as an early diverging

281 species in Iurida based on transcriptome analyses (Sharma et al. 2015), highlights the need for

282 further investigation using genome wide markers for this ancient group.

284 scorpions indicate that our probe set will have applications beyond deep-level systematics and

285 taxonomy. The UCE loci show promise for use in species-level phylogenetics and species

286 delimitation because the flanking regions show increasing variability as distance from the core

287 UCE increases (Fig. 3). UCEs enrichment produced 480 loci with more than 8,000 variable sites

288 across two members of the Antrodiaetus riversi complex, 292 loci with more than 25,000

289 variable sites across two harvestmen species in the genus Briggsus, and 585 loci with more than

2907,000 variable sites across an individual of the scorpion genus Centruroides and the published

291 Centruroides genome (Table 3). These patterns of UCE variability are correlated with

292 divergence in mitochondrial cytochrome oxidase I (Fig. 4). The proportion of polymorphic loci

293 (minimum 0.9) and average variable sites per polymorphic locus (minimum 14) seen across these

294 pairs is greater than those reported in the study of Smith et al. (2014; maximum 0.77 and 3.2,

295 respectively), which demonstrated the utility of UCEs at shallow time scales for species-level

296 phylogenetic and species delimitation analyses in birds. UCEs may be advantageous for shallow-

297 level studies in Arachnida because other techniques, such as RADseq, are susceptible to locus

298 dropout in arachnid species with relatively deep levels of divergence (Bryson et al. 2016;

299 Derkarabetian et al. in press). Our UCE probe set will also provide a complementary/alternative

300 resource to the recently developed spider anchored-enrichment loci, which were used to produce 
301 a comparable number of loci (455) and a well-resolved phylogeny for the North American

302 tarantula genus Aphonopelma (Hamilton et al. 2016).

303 The arachnid-specific UCEs have utility and phylogenetic informativeness at all levels of

304 Arachnida, spanning extremely ancient divergence times between orders (at least 400 MYA) to

305 more recent congeneric divergences (<10 MYA for Antrodiaetus, Hedin et al. 2012). Thus, these

306 markers will be an integral component of future comparative studies. Arachnids are an extremely

307 diverse group, and yet it is estimated that less than half of arachnid species have been formally

308 described (Chapman 2009). Species delimitation increasingly relies on phylogenomic data that

309 can be sampled consistently across diverse taxa (Leaché et al. 2014; Smith et al., 2014; Rannala

310 2015; Harvey et al. 2016). The presence of UCEs across Arachnida makes these a valuable

311 resource for discovery of new species and for inferring phylogenetic relationships in

312 understudied arachnid groups.

\section{Acknowledgements}

315 For help during lab work we thank Carl Oliveros and Rich Harrington. John Klicka provided

316 other assistance. Tim Burkhart, Ken MacNeil, Peter Clausen, and Cor Vink provided assistance

317 with specimen collection. Live specimen photos were provided by Mardon Erbland and Warren

318 E. Savary. Funding for this research was provided by NSF grant DEB-1354558 to MH. Startup

319 funds from LSU and DEB-1242260 to BCF supported computational portions of this work.

\section{Data Availability}

321 Raw sequence reads are available in the NCBI Short Read Archive accession SRP078995

322 (BioProject ID \#PRJNA328972), and untrimmed contigs have been given accessions \#\#\#\#\#- 
323 \#\#\#\#\#. Trimmed alignments used for phylogenetic analyses are available in Dryad (doi: \#\#\#\#\#).

\section{Author Contributions}

325 All authors contributed to the conception and design of the experiments; JS, SD, MH, RWB

326 contributed samples; JS, SD, RWB, BCF conducted lab work; JS, SD, BCF analyzed data; all 327 authors edited and commented on the manuscript.

329 References

330 Alexander RP, Fang G, Rozowsky J, Snyder M, Gerstein MB (2010) Annotating non-coding 331 regions of the genome. Nature Reviews Genetics, 11, 559-571.

332 Bejerano G, Pheasant M, Makunin I, Stephen S, Kent WJ, Mattick JS, Haussler D (2004)

333 Ultraconserved elements in the human genome. Science, 304, 1321-1325.

334 Bond JE, Garrison NL, Hamilton CA, Godwin RL, Hedin M, Agnarsson I (2014) Phylogenomics

335 resolves a spider backbone phylogeny and rejects a prevailing paradigm for orb web

336 evolution. Current Biology, 24, 1765-1771. doi.org/10.1016/j.cub.2014.06.034.

337 Bryson RW, Savary WE, Zellmer AJ, Bury RB, McCormack JE (2016) Genomic data reveal

338 ancient microendemism in forest scorpions across the California Floristic Province.

339 Molecular Ecology, doi: 10.1111/mec.13707.

340 Castresana J (2000) Selection of conserved blocks from multiple alignments for their use in 341 phylogenetic analysis. Molecular Biology and Evolution, 17, 540-552.

342 Chapman AD (2009) Numbers of Living Species in Australia and the World, (2nd edn), $343 \quad$ Australian Biodiversity Information Services. 
344 Crawford NG, Faircloth BC, McCormack JE, Brumfield RT, Winker K, Glenn TC (2012) More than 1000 ultraconserved elements provide evidence that turtles are the sister group of archosaurs. Biology Letters, 8, 783-786. doi:10.1098/rsb1.2012.0331.

Derkarabetian S, Burns M, Starrett J, Hedin M. (2016) Population genomic evidence for multiple refugia in montane-restricted harvestmen (Arachnida, Opiliones, Sclerobunus robustus) from the southwestern United States. Molecular Ecology, In Press.

Derti A, Roth FP, Church GM, Wu C (2006) Mammalian ultraconserved elements are strongly depleted among segmental duplications and copy number variants. Nature Genetics, $\mathbf{3 8}$,

Dunlop JA (1996) A trigonotarbid arachnid from the Upper Silurian of Shropshire.

$$
\text { 1216-1220. doi:10.1038/ng1888 }
$$

Dunlop JA, Tetlie OE, Prendini L (2008) Reinterpretation of the Silurian scorpion Proscorpius osborni (Whitfield): integrating data from Palaeozoic and Recent scorpions.

Faircloth BC (2016) Identifying conserved genomic elements and designing universal probe sets to enrich them. Molecular Ecology Resources, submitted.

Faircloth BC, Branstetter MG, White ND, Brady SG (2015) Target enrichment of ultraconserved elements from arthropods provides a genomic perspective on relationships among Hymenoptera. Molecular Ecology Resources, 15, 489-501. doi: 10.1111/1755-

364 Faircloth BC, McCormack JE, Crawford NG, Harvey MG, Brumfield RT, Glenn TC (2012) 
evolutionary timescales. Systematic Biology, 61, 717-726. DOI:10.1093/sysbio/sys004.

367

368

Faircloth BC, Sorenson L, Santini F, Alfaro ME (2013) A phylogenomic perspective on the radiation of ray-finned fishes based upon targeted sequencing of ultraconserved elements (UCEs). PLoS ONE 8, e65923. doi:10.1371/journal.pone.0065923.

Fernández R, Hormiga G, Giribet G (2014) Phylogenomic analysis of spiders reveals nonmonophyly of orb weavers. Current Biology, 24, 1772-1777. doi.org/10.1016/j.cub.2014.06.035.

Garrsion NL, Rodriguez J, Agnarsson I, Coddington JA, Griswold CE, Hamilton CA, Hedin M, Kocot KM, Ledford JM, Bond JE (2016) Spider phylogenomics: untangling the spider tree of life. PeerJ, 4:e1719; DOI 10.7717/peerj.1719.

Gilbert PS, Chang J, Pan C, Sobel EM, Sinsheimer JS, Faircloth BC, Alfaro ME (2015) Genome-wide ultraconserved elements exhibit higher phylogenetic informativeness than traditional gene markers in percomorph fishes. Molecular Phylogenetics and Evolution, 92, 140-146. doi.org/10.1016/j.ympev.2015.05.027.

Giribet G, Edgecombe GD, Wheeler WC, Babbitt C (2002) Phylogeny and systematic position of opiliones: A combined analysis of chelicerate relationships using morphological and molecular data1. Cladistics, 18, 5-70.

Glenn TC, Nilsen R, Kieran TJ, Finger JW, Pierson TW, Bentley KE, et al. (2016) Adapterama I: Universal stubs and primers for thousands of dual-indexed Illumina libraries (iTru \& iNext). bioRxiv, doi: 10.1101/049114.

Grabherr MG, Haas BJ, Yassour M, Levin JZ, Thompson DA, Amit I, Adiconis X, Fan L, Raychowdhury R, Zeng Q, Chen Z, Mauceli E, Hacohen N, Gnirke A, Rhind N, di Palma 

transcriptome assembly from RNA-Seq data without a reference genome. Nature Biotechnology, 29, 644-652.

Hamilton CA, Hendrixson BE, Bond JE (2016) Taxonomic revision of the tarantula genus Aphonopelma Pocock, 1901 (Araneae, Mygalomorphae, Theraphosidae) within the United States. ZooKeys, 560, 1.

Harvey MG, Smith BT, Glenn TC, Faircloth BC, Brumfield RT (2016) Sequence Capture Versus Restriction Site Associated DNA Sequencing for Shallow Systematics. Systematic

Harvey MS (2002) The neglected cousins: What do we know about the smaller arachnid orders? Journal of Arachnology, 30, 357-372.

Hedin M, Starrett J, Akhter S, Schönhofer AL, Shultz JW (2012) Phylogenomic resolution of paleozoic divergences in harvestmen (Arachnida, Opiliones) via analysis of nextbiogeographic patterns revealed in the genetic history of low-dispersal mygalomorph

Jeram AJ, Selden PA, Edwards D (1990) Land animals in the Silurian: arachnids and myriapods from the Shropshire, England. Science, 250, 658-661. 780. 
Leaché AD, Fujita MK, Minin VN, Bouckaert RR (2014) Species Delimitation using genomewide SNP data. Systematic Biology. 63, 534-542. doi:10.1093/sysbio/syu018.

412 Masta SE, Longhorn SJ, Boore JL (2009) Arachnid relationships based on mitochondrial genomes: asymmetric nucleotide and amino acid bias affects phylogenetic analyses.

Laurie M (1899) On a Silurian scorpion and some additional eurypterid remains from the Pentland Hills. Transactions of the Royal Society of Edinburgh, 39, 575-590.

Manthey JD, Campillo LC, Burns KJ, Moyle RG (2016) Comparison of target-capture and restriction-site associated DNA sequencing for phylogenomics: a test in cardinalid

McCormack JE, Faircloth BC, Crawford NG, Gowaty PA, Brumfield RT, Glenn TC (2012) Ultraconserved elements are novel phylogenomic markers that resolve placental mammal phylogeny when combined with species-tree analysis. Genome Research, 22, 746-754.

McCormack JE, Tsai WLE, Faircloth (2015) Sequence capture of ultraconserved elements from bird museum specimens. Molecular Ecology Resources, 1-15. doi: 10.1111/1755evolutionary radiation using ultraconserved elements (UCEs): Evidence for a bias in some multispecies coalescent methods. Systematic Biology, 65, 612-627. 
432 Rannala B (2015) The art and science of species delimitation. Current Zoology. 61, 846-853.

433 Regier JC, Shultz JW, Zwick A, Hussey A, Ball B, Wetzer R, Martin JW, Cunningham CW

434 (2010) Arthropod relationships revealed by phylogenomic analysis of nuclear protein-

435 coding sequences. Nature, 463, 1079-1083. doi:10.1038/nature08742.

436 Rehm P, Pick C, Borner J, Markl J, Burmester T (2012) The diversity and evolution of

437 chelicerate hemocyanins. BMC Evolutionary Biology, 12, 19.

438 Rota-Stabelli O, Daley AC, Pisani D (2013) Molecular timetrees reveal a Cambrian colonization

439 of land and a new scenario for ecdysozoan evolution. Current Biology, 23, 392-398.

440 Sharma PP, Giribet G (2014) A revised dated phylogeny of the arachnid order Opiliones.

441 Frontiers in Genetics, 5, 1-13. doi:10.3389/fgene.2014.00255.

442 Sharma PP, Fernández R, Esposito LA, González-Santillán E, Monod L (2015) Phylogenomic

443 resolution of scorpions reveals multilevel discordance with morphological phylogenetic

$444 \quad$ signal. Proceedings of the Royal Society of London B, 282, 20142953.

$445 \quad$ doi.org/10.1098/rspb.2014.2953.

446 Sharma PP, Kaluziak ST, Pérez-Porro AR, González VL, Hormiga G, Wheeler WC, Giribet G

447 (2014) Phylogenomic interrogation of arachnida reveals systematic conflicts in

448 phylogenetic signal. Molecular Biology and Evolution, 31, 2963-2984.

449 doi:10.1093/molbev/msu235.

450 Shultz JW (2007) A phylogenetic analysis of the arachnid orders based on morphological

451 characters. Zoological Journal of the Linnean Society, 150, 221-265.

$452 \quad$ DOI:10.1093/sysbio/syt061.

453 Smith BT, Harvey MG, Faircloth BC, Glenn TC, Brumfield RT (2014) Target capture and 
massively parallel sequencing of ultraconserved elements for comparative studies at shallow evolutionary time scales. Systematic Biology, 63, 83-95.

456 Stamatakis A, Hoover P, Rougemont J (2008) A rapid bootstrap algorithm for the RAxML web servers. Systematic biology, 57, 758-771.

458 Stamatakis A (2014) RAxML version 8: a tool for phylogenetic analysis and post-analysis of large phylogenies. Bioinformatics, 30, 1312-1313.

460 Streicher JW, Schulte II JA, Weins JJ (2016) How should genes and taxa be sampled for phylogenomic analyses with missing data? An empirical study in Iguanian lizards. Systematic Biology, 65, 128-145. DOI:10.1093/sysbio/syv058.

463 Talavera G, Castresana J (2007) Improvement of phylogenies after removing divergent and ambiguously aligned blocks from protein sequence alignments. Systematic biology, 56,

466 Wheeler WC, Hayashi CY (1998) The phylogeny of the extant chelicerate orders. Cladistics, 14, 173-192.

468 Zhang Z-Q (2011) Phylum Arthropoda von Siebold, 1848 In: Zhang, Z.-Q. (Ed.) Animal 469 biodiversity: An outline of higher-level classification and survey of taxonomic richness. Zootaxa, 3148, 99-103. 
Tables

Table 1. Sample information and sequencing statistics. DNA refers to starting quantity that was processed. Voucher specimens are housed in the San Diego State University Terrestrial Arthropod Collection (SDSU_TAC).

\begin{tabular}{|c|c|c|c|c|c|c|c|c|c|}
\hline Order & Species & Voucher & DNA (ng) & Pool & Raw Read Pairs & Reads Pass QC & \% Pass QC & Contigs & UCE loci \\
\hline Acariformes & Neomolgus littoralis & AR014 & 500 & G & 633368 & 595582 & 94.03 & 26192 & 170 \\
\hline Amblypygi & Damon diadema & AR010 & 500 & $\mathrm{~F}$ & 1623520 & 1484780 & 91.45 & 26761 & 442 \\
\hline Araneae & Antrodiaetus riversi & AR17 & 494 & $A$ & 1277026 & 1216925 & 95.29 & 39879 & 576 \\
\hline Araneae & Antrodiaetus riversi & MY1253 & 360 & $A$ & 1240653 & 1171503 & 94.43 & 28151 & 571 \\
\hline Araneae & Calisoga sp. & MY4085 & 500 & $A$ & 1283059 & 1209706 & 94.28 & 37800 & 664 \\
\hline Araneae & Diguetia signata & G0580 & 500 & $\mathrm{C}$ & 2109935 & 1988677 & 94.25 & 76172 & 504 \\
\hline Araneae & Euagrus chisoseus & MY4295 & 500 & $A$ & 1672688 & 1592368 & 95.20 & 46272 & 674 \\
\hline Araneae & Habronattus tarsalis & HA0955 & 500 & $\mathrm{C}$ & 1016446 & 957507 & 94.20 & 30683 & 484 \\
\hline Araneae & Hebestatis theveneti & MY4392 & 500 & $\mathrm{~A}$ & 1327914 & 1257588 & 94.70 & 34651 & 722 \\
\hline Araneae & Hypochilus pococki & H0595 & 500 & $\mathrm{C}$ & 1143368 & 1081293 & 94.57 & 32241 & 552 \\
\hline Araneae & Kukulcania sp. & G0551 & 500 & $\mathrm{C}$ & 1032516 & 984284 & 95.33 & 32448 & 466 \\
\hline Araneae & Liphistius malayanus & MIS1 & 500 & A & 832843 & 781359 & 93.82 & 26169 & 557 \\
\hline Araneae & Megahexura fulva & MY4378 & 500 & $A$ & 1680701 & 1602923 & 95.37 & 60357 & 565 \\
\hline Araneae & Nesticus gertschi & N0806 & 456 & B & 1354456 & 1238198 & 91.42 & 19918 & 543 \\
\hline Araneae & Oecobius navus & GAR4 & 280 & $\mathrm{C}$ & 1596815 & 1523543 & 95.41 & 39711 & 539 \\
\hline Araneae & Porrhothele sp. & MY0857 & 500 & A & 1121499 & 1046279 & 93.29 & 33797 & 628 \\
\hline Opiliones & Bishopella laciniosa & OP0569 & 500 & $\mathrm{D}$ & 1486862 & 1385611 & 93.19 & 42527 & 329 \\
\hline Opiliones & Briggsus bilobatus & OP3559 & 216 & $\mathrm{E}$ & 1148872 & 1093882 & 95.21 & 26325 & 406 \\
\hline Opiliones & Briggsus pacificus & OP3625 & 320 & $\mathrm{D}$ & 1355200 & 1267279 & 93.51 & 50366 & 399 \\
\hline Opiliones & Fumontana deprehendor & OP0623 & 432 & $\mathrm{D}$ & 1265798 & 1174121 & 92.76 & 28841 & 356 \\
\hline Opiliones & Leiobunum calcar & OP1089 & 500 & $\mathrm{D}$ & 1315511 & 1242038 & 94.41 & 47403 & 346 \\
\hline Opiliones & Metanonychus $n$. nigricans & OP3704 & 247 & $\mathrm{D}$ & 1313727 & 1227411 & 93.43 & 43275 & 377 \\
\hline Opiliones & Sabacon cavicolens & OP1518 & 500 & $\mathrm{D}$ & 990843 & 907152 & 91.55 & 18060 & 293 \\
\hline Opiliones & Siro sp. & OP3383 & 108 & $\mathrm{D}$ & 1260358 & 1179969 & 93.62 & 33373 & 369 \\
\hline Scorpiones & Bothriurus keyserlingi & AR021 & 500 & 1 & 1238117 & 1183837 & 95.62 & 23438 & 500 \\
\hline Scorpiones & Centruroides sculpturatus & AR015 & 500 & $\mathrm{H}$ & 3913560 & 3750267 & 95.83 & 100622 & 706 \\
\hline Scorpiones & Diplocentrus peloncillensis & AR016 & 500 & $\mathrm{H}$ & 533530 & 507517 & 95.12 & 14225 & 402 \\
\hline Scorpiones & Hadrurus arizonensis & AR017 & 500 & $\mathrm{H}$ & 734122 & 700561 & 95.43 & 17206 & 565 \\
\hline Scorpiones & Paravaejovis spinigerus & AR018 & 500 & $\mathrm{H}$ & 1035158 & 990302 & 95.67 & 22872 & 478 \\
\hline \multirow[t]{2}{*}{ Thelyphonida } & Mastigoproctus giganteus & AR011 & 500 & $\mathrm{~F}$ & 926004 & 871445 & 94.11 & 22195 & 431 \\
\hline & & & & Mean & 1315482.3 & 1240463.57 & 94.22 & 36064.33 & 487.13 \\
\hline
\end{tabular}


Table 2. Matrix statistics. PI = parsimony informative sites.

\begin{tabular}{ll|llllll} 
& & \multicolumn{7}{|c}{$\mathbf{5 0 \%}$ taxon coverage } \\
Dataset & N & Loci & Length & $\begin{array}{l}\text { Mean } \\
\text { Length }\end{array}$ & $\begin{array}{l}\text { Min-Max } \\
\text { length }\end{array}$ & PI & \% PI \\
\hline 2per Arachnid & 11 & 602 & 199,810 & 331.91 & $113-853$ & 60,468 & 30.3 \\
UCEsample & 32 & 510 & 105,337 & 206.54 & $80-746$ & 49,362 & 46.9 \\
Araneae +OUT & 20 & 686 & 169,134 & 246.55 & $92-833$ & 67,644 & 40.0 \\
Araneae & 19 & 724 & 181,915 & 251.26 & $92-833$ & 71,786 & 39.5 \\
Opiliones +OUT & 9 & 435 & 158,314 & 363.94 & $97-893$ & 33,263 & 21.0 \\
Opiliones & 8 & 381 & 153,187 & 402.07 & $97-914$ & 29,807 & 19.5 \\
Scorpiones+OUT & 8 & 627 & 318,178 & 507.46 & $145-1136$ & 65,227 & 20.5 \\
Scorpiones & 7 & 749 & 452,309 & 603.88 & $99-1141$ & 64,892 & 14.4 \\
\hline Mean & & $\mathbf{5 8 9 . 3}$ & $\mathbf{2 1 7 2 7 3}$ & $\mathbf{3 6 4 . 2}$ & & $\mathbf{5 5 3 0 6 . 1}$ & $\mathbf{2 9 . 0}$ \\
\hline
\end{tabular}

Table 3. Congeneric matrix statistics.

\begin{tabular}{|c|c|c|c|c|c|c|c|c|c|}
\hline Dataset & Loci & Total Length & Mean Length & Min-Max length & Variable Sites & $\%$ variable & $\begin{array}{l}\# \\
\text { Polymorp } \\
\text { hic Loci }\end{array}$ & $\begin{array}{l}\text { Proportio } \\
\text { n } \\
\text { Polymorp } \\
\text { hic }\end{array}$ & $\begin{array}{l}\text { Average } \\
\text { variable } \\
\text { sites per } \\
\text { variable } \\
\text { locus }\end{array}$ \\
\hline Antrodiaetus & 480 & 329,110 & 685.65 & $129-1248$ & 8,389 & 2.55 & 476 & 0.99 & 17.62 \\
\hline Briggsus & 292 & 172,562 & 590.97 & $144-1190$ & 25,524 & 14.79 & 292 & 1.00 & 87.41 \\
\hline Centruroides & 585 & 583,454 & 997.36 & $243-1164$ & 7,660 & 1.31 & 527 & 0.90 & 14.54 \\
\hline
\end{tabular}



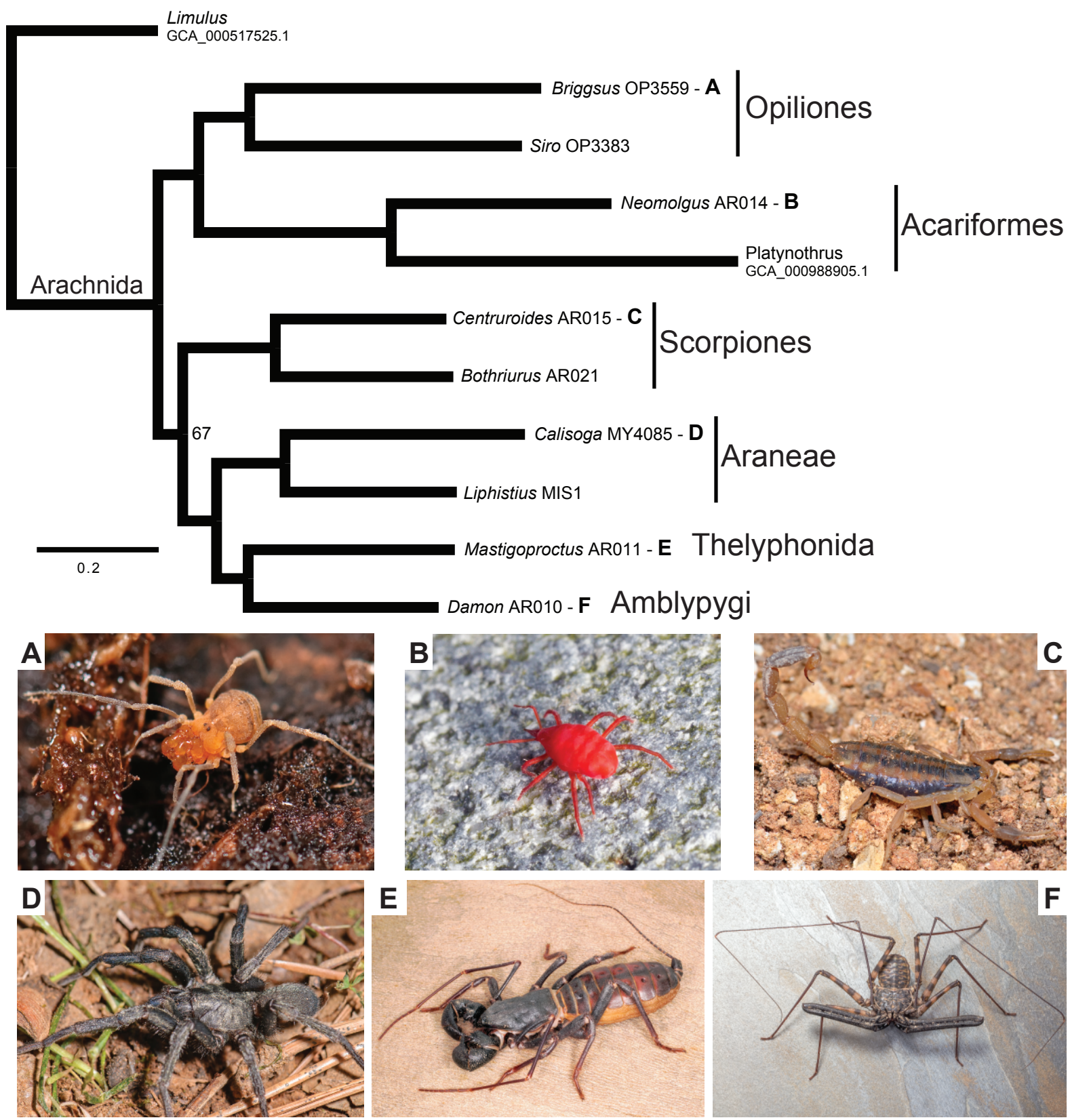

Figure 1. Maximum likelihood phylogeny based on 50\% minimum taxon coverage "2perArachnid" dataset, including representative images of included arachnid orders. Nodes have $100 \%$ bootstrap support, unless otherwise indicated. Letters A-F correspond to

488 representative images of included arachnid orders. Photo credit: M Hedin (A, C, D), M Erbland 489 (B), WE Savary (E, F). 
bioRxiv preprint doi: https://doi.org/101101/065201. this version posted July 22 2016. The copyright holder for this preprint (which was not certified by peer review) is the author/funder, who has granted bioRxiv a license to display the preprint in perpetuity. It is made available under aCC-BY-NC-ND 4.0 International license.

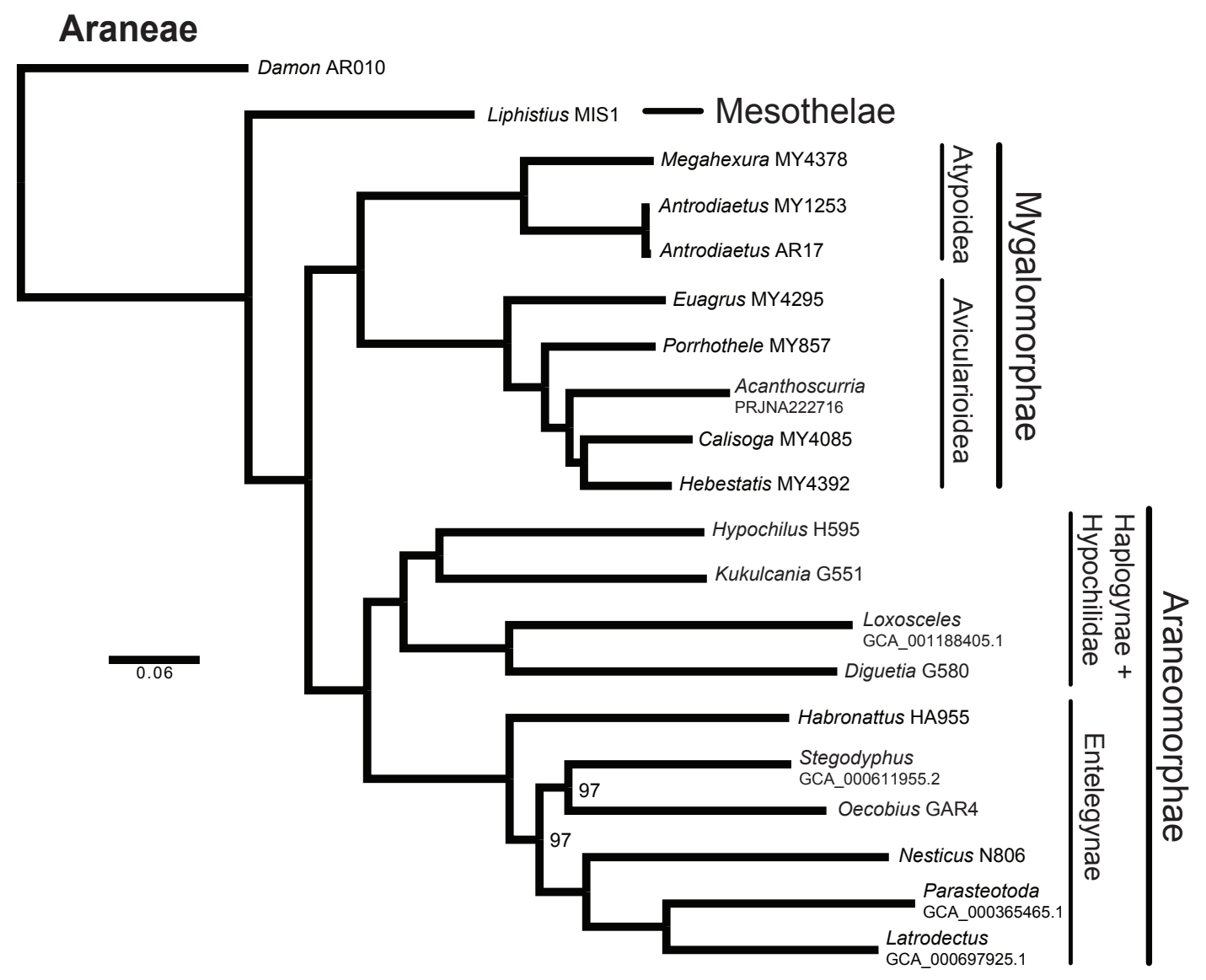

Figure 2. Maximum likelihood phylogeny based on 50\% minimum taxon coverage dataset for the Araneae+OUT dataset. Nodes have 100\% bootstrap support, unless otherwise indicated. 
bioRxiv preprint doi: https://doi.org/10.1101/065201. this version posted July 22 2016. The copyright holder for this preprint (which was not certified by peer review) is the author/funder, who has granted bioRxiv a license to display the preprint in perpetuity. It is made available under aCC-BY-NC-ND 4.0 International license.

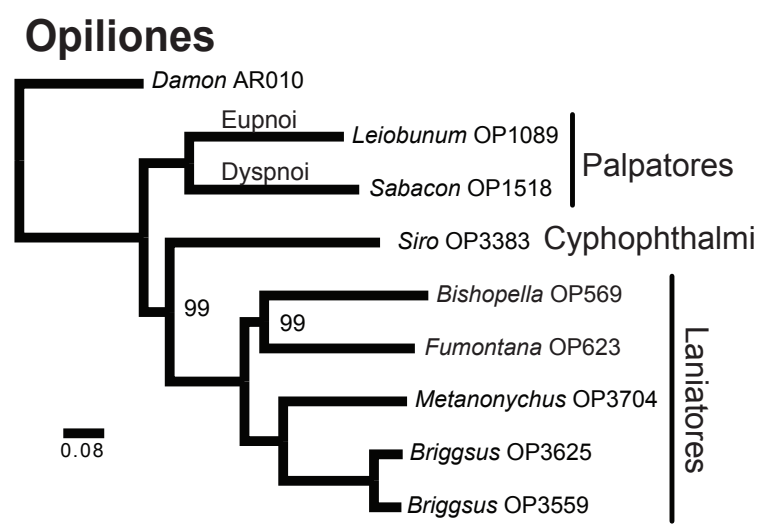

\section{Scorpiones}

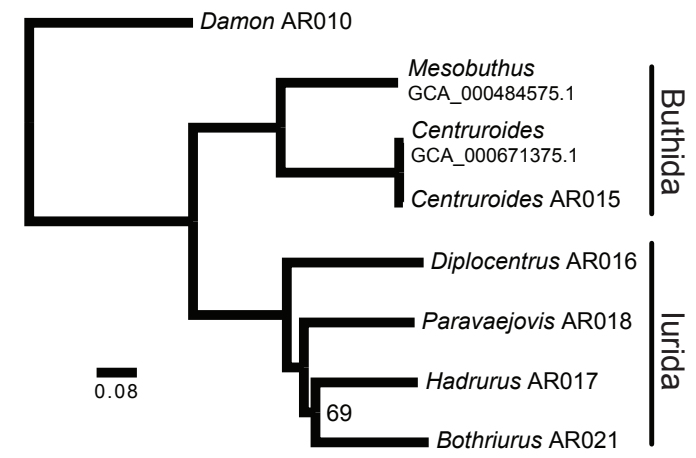

Figure 3. Maximum likelihood phylogenies based on 50\% minimum taxon coverage dataset for the Opiliones+OUT and Scorpiones+OUT datasets. Nodes have 100\% bootstrap support, unless otherwise indicated. 

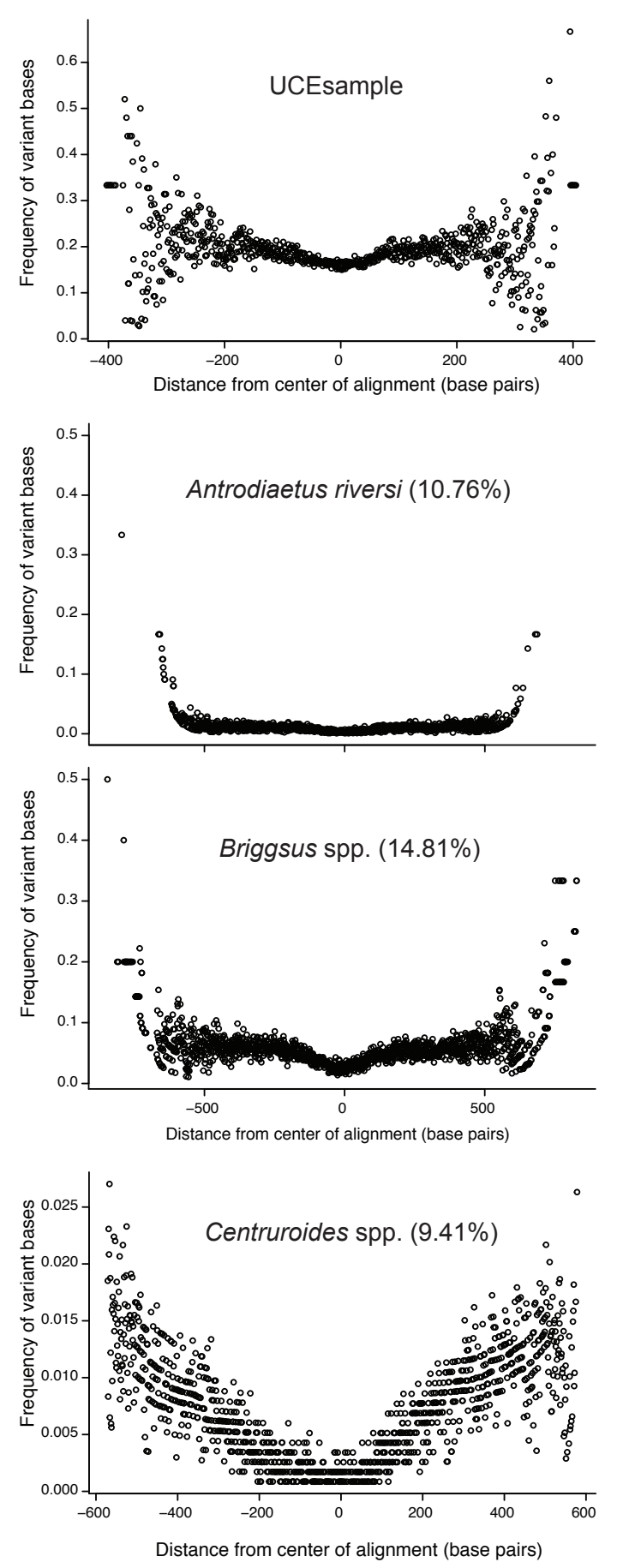

Figure 4. Variability increases as distance from core UCE increases. Data points with no variability were removed. For Antrodiaetus (turret spiders) and Briggsus (harvestmen) comparisons, the scale of x-axis is identical. Numbers in parentheses are uncorrected COI divergence values. In Antrodiaetus, an outlier was removed for better viewing. Cytochrome oxidase subunit I (COI) distances for Centruroides (bark scorpions) calculated from GenBank accessions AY995831.1 (C. sculpturatus) and AY995833.1 (C. exilicauda). 